\title{
Simple Graphical Selection of Optical Materials for an Athermal and Achromatic Design Using Equivalent Abbe Number and Thermal Glass Constant
}

\author{
Young-Ju Kim, Yeong-Sik Kim, and Sung-Chan Park* \\ Department of Physics, Dankook University, Cheonan 330-714, Korea
}

(Received January 9, 2015 : accepted March 23, 2015)

\begin{abstract}
This paper presents a new graphical method for selecting a pair of optical glasses to simultaneously achromatize and athermalize an imaging lens made of materials in contact. An athermal glass map that plots thermal glass constant versus inverse Abbe number is derived through analysis of optical glasses and plastic materials in visible light. By introducing the equivalent Abbe number and equivalent thermal glass constant, although it is a multi-lens system, we have a simple way to visually identify possible optical materials. Applying this method to design a phone camera lens equipped with quarter inch image sensor having 8-mega pixels, the thermal defocuses over $-20^{\circ} \mathrm{C}$ to $+60^{\circ} \mathrm{C}$ are reduced to be much less than the depth of focus of the system.
\end{abstract}

Keywords: Athermalization, Achromatization, Lens design, First orders

OCIS codes : (220.3620) Lens system design; (220.0220) Optical design and fabrication; (160.4670) Optical Materials

\section{INTRODUCTION}

In recent optical instruments such as mobile phone and black box cameras, plastic materials are widely used. Because of their inherent temperature sensitivity, however, variation in ambient temperature significantly induces a change of refractive index, curvature of lens, and thickness. Since the thermal defocus caused by such changes degrades the image quality, a lens system using plastics should be designed to have stable performance over the specified temperature range.

To reduce thermal and chromatic errors, many design methods have been reported [1-8]. Perry studied the effect of temperature changes on optical performance in infrared optics. Rogers showed that the multi-lens system could be achromatized and athermalized with three materials by solving three equations, but it was difficult to find a proper combination of materials [5].

As alternative approaches to overcome these problems, graphic methods have been developed. Rayces and Lebich proposed a $\gamma \mathrm{V}-\mathrm{V}$ diagram and showed that chromatic aberration corresponds to the area of a triangle [6]. Tamagawa, et al. suggested other graphical approaches by plotting the thermal dispersive power versus chromatic dispersive power and included a discussion of selecting the element powers in addition to the element materials [7, 8]. Although this method is robust, it requires complex nomographs and calculations to achieve an achromatized and athermalized system having more than three elements. This research has been performed mainly on infrared optics, and there are few studies on visible optics.

In order to solve these difficulties, in this paper we introduce the equivalent Abbe number and equivalent thermal glass constant. Even though a lens system is composed of many elements, we can simply identify a pair of materials that satisfies the athermal and achromatic conditions, by selecting the corresponding materials for an equivalent lens from an athermal glass map. By application of the concept of equivalence to the phone camera lens composed of four elements, a good solution having small chromatic and thermal defocus is obtained. The chromatic error is $\pm 10 \mu \mathrm{m}$ between $\mathrm{C}$ and F-lines, and the thermal defocuses over $-20^{\circ} \mathrm{C}$ to $+60^{\circ} \mathrm{C}$ are found to be much less than the depth of focus of the system. This lens is consequently achromatized and passively athermalized.

\footnotetext{
*Corresponding author: scpark@dankook.ac.kr
} 


\section{ATHERMAL AND ACHROMATIC CONDITIONS}

\subsection{Achromatized Lens}

For a single thin lens, the change in optical power $(\Delta \phi)$ due to a wavelength change is given by

$$
\Delta \phi=\phi_{F}-\phi_{C}=\frac{\phi_{d}}{v_{d}}
$$

where $v_{d}$ is the Abbe number at d-line.

The analogous equation quantifying the power change of a doublet with wavelength is given by

$$
\begin{aligned}
& \Delta \phi=\phi_{F}-\phi_{C}=\Delta \phi_{1}+\Delta \phi_{2}, \\
& =\frac{\phi_{1}}{v_{1}}+\frac{\phi_{2}}{v_{2}}
\end{aligned}
$$

For a doublet to be achromatic, each element is required to have power as follows:

$$
\phi_{1}=\left(\frac{v_{1}}{v_{1}-v_{2}}\right) \phi, \quad \phi_{2}=-\left(\frac{v_{2}}{v_{1}-v_{2}}\right) \phi .
$$

As a result, by selecting proper Abbe numbers in Eq. (3), an achromatic system can be realized.

\subsection{Athermalized Lens}

The variations of temperature change the radius of curvature, lens thickness and air spacing, and refractive index. These changes cause alignment errors and degrade the optical performance. An athermal system is meant to have stable optical performance, even during temperature changes in the optical system [9].

Thermal defocus is the change in the focus position on axis with temperature changes due to the variation of the index with temperature and the thermal expansion of the material. Table 1 lists the quantitative variations of design parameters due to temperature change [2].

In Table 1, the parameters of $n(T), R(T), t(T)$, and $L(T)$ are measured values after temperature change. The coefficients of a linear thermal expansion(CTE) are designated as $\alpha_{g}$ for the lens material and $\alpha_{m}$ for the housing material,

TABLE 1. Variations of design parameters due to temperature change

\begin{tabular}{c|l}
\hline \hline Index & $n(T)=n+d n=n+(d n / d T) \Delta T$ \\
\hline Radius & $R(T)=R+d R=R\left(1+\alpha_{g} \Delta T\right)$ \\
\hline Thickness & $t(T)=t+d t=t\left(1+\alpha_{g} \Delta T\right)$ \\
\hline Space & $L(T)=L+d L=L\left(1+\alpha_{m} \Delta T\right)$ \\
\hline Air & $n_{\text {air }}(T)=n_{\text {air }}+d n_{\text {air }}=n_{\text {air }}+\left(d n_{\text {air }} / d T\right) \Delta T$ \\
\hline
\end{tabular}

respectively. The parameter $d n / d T$ denotes the variation of the refractive index with temperature.

The change in optical power of a single thin lens due to a temperature change is given by

$$
\begin{aligned}
& \frac{d \phi}{d T}=\frac{d n}{d T}\left(c_{1}-c_{2}\right)+(n-1)\left(-\frac{1}{r_{1}^{2}} \frac{d r_{1}}{d T}+\frac{1}{r_{2}^{2}} \frac{d r_{2}}{d T}\right), \\
& =\phi\left(\frac{1}{n-1} \frac{d n}{d T}-\alpha\right), \\
& =\gamma \phi
\end{aligned}
$$

where,

$\alpha \equiv \frac{1}{r_{1}} \frac{d r_{1}}{d T}=\frac{1}{r_{2}} \frac{d r_{2}}{d T}:$ Coefficient of linear thermal expansion (CTE), $\gamma=\frac{1}{n-1} \frac{d n}{d T}-\alpha$ : Thermal glass constant.

From Eq. (4), The change in optical power of a single thin lens is proportional to the thermal glass constant $(\gamma)$. Thus, if the thermal glass constants are known, the thermal defocus can be estimated for a steady temperature change. This concept would apply to the optical system composed of many elements. Similarly, the change in optical power of a doublet due to a temperature change is

$$
\frac{d \phi}{d T}=\gamma_{1} \phi_{1}+\gamma_{2} \phi_{2}
$$

Inserting the achromatic condition of Eq. (3) into Eq. (5) results in an expression for the athermal and achromatic conditions for a doublet, as follows:

$$
\gamma_{1} v_{1}-\gamma_{2} v_{2}=0
$$

where $\gamma_{1}$ and $\gamma_{2}$ are the thermal glass constants for each lens.

\section{ATHERMAL AND ACHROMATIC DESIGN USING EQUIVALENT ABBE NUMBER AND THERMAL GLASS CONSTANT}

By requiring a system to satisfy the equations for total power, achromatism, and athermalism, the athermal and achromatic solution can be found. For a system with an arbitrary number of elements, the following equations must be satisfied [10]:

$$
\begin{aligned}
& \text { Total power : } \sum \phi_{i}=\phi \\
& \text { Achromatism : } \Delta \phi=\phi_{F}-\phi_{C}=\sum \frac{\phi_{i}}{V_{i}}=0 \\
& \text { Athermalism : } \frac{d \phi}{d T}=\sum \gamma_{i} \phi_{i}=0
\end{aligned}
$$


All of these equations assume thin lenses in contact with each other. Although this is not a physically realizable solution, these equations can provide a good starting point. In the strictest sense, Equation (9) may include a term for thermal expansion of a housing material. However, the contribution of this term is so small compared to that of the material's $d n / d T$ in a short back focal system, as will be discussed in Section IV, that it has not been included here.

An athermal and achromatic doublet can be simply obtained from Eq. (6), however, it requires a very complicated process to have a lens system with many elements being athermal and achromatic simultaneously [7, 8]. In this study, the equivalent Abbe number $\left(v_{e f f}\right)$ and equivalent thermal glass constant $\left(\gamma_{\text {eff }}\right)$ are introduced to select a pair of glasses on an athermal glass map, in order to achieve simply an athermal and achromatic system like a doublet.

For a given optical power, Equation (1) gives an expression for Abbe number:

$$
v_{d}=\frac{\phi_{d}}{\phi_{F}-\phi_{C}}
$$

Since the total power of a doublet is $\phi=\phi_{1}+\phi_{2}$, inserting Eq. (2) into Eq. (10) results in an equivalent Abbe number $\left(v_{d o u}\right)$ of a doublet, as follow:

$$
v_{\text {dou }}=\frac{\phi_{1}+\phi_{2}}{\left(\phi_{1} / v_{1}\right)+\left(\phi_{2} / v_{2}\right)}
$$

Although two materials have different Abbe numbers, it can be defined as one valued Abbe number that is referred to as an equivalent Abbe number. In the same way, the equivalent Abbe number of a triplet thin lens in contact is given by

$$
v_{t r i}=\frac{\phi_{1}+\phi_{2}+\phi_{3}}{\left(\phi_{1} / v_{1}\right)+\left(\phi_{2} / v_{2}\right)+\left(\phi_{3} / v_{3}\right)}
$$

Extending this method to an $\mathrm{N}$-elements system in contact, the equivalent Abbe number of a system is expressed as follows:

$$
v_{e f f}=\frac{\sum_{i=1}^{N} \phi_{i}}{\sum_{i=1}^{N}\left(\phi_{i} / v_{i}\right)}
$$

In common with equivalent Abbe number, equivalent thermal glass constant can be defined for a system with many elements. From Eqs. (4) and (5), equivalent thermal glass constant $\left(\gamma_{d o u}\right)$ of a doublet is given by

$$
\gamma_{\text {dou }}=\frac{\gamma_{1} \phi_{1}+\gamma_{2} \phi_{2}}{\phi_{1}+\phi_{2}}
$$

Also, the equivalent thermal glass constants of a triplet and an $\mathrm{N}$-elements system in contact are respectively given by

$$
\begin{aligned}
& \gamma_{t r i}=\frac{\gamma_{1} \phi_{1}+\gamma_{2} \phi_{2}+\gamma_{3} \phi_{3}}{\phi_{1}+\phi_{2}+\phi_{3}}, \\
& \gamma_{e f f}=\frac{\sum_{i=1}^{N} \gamma_{i} \phi_{i}}{\sum_{i=1}^{N} \phi_{i}} .
\end{aligned}
$$

Although a system has an arbitrary number of elements, from Eqs. (13) and (16), Abbe number and thermal glass constant of a system can be simply obtained. Consequently, a pair of materials that satisfy the Eq. (6) can graphically be selected to simultaneously achromatize and passively athermalize an imaging lens. In this paper, the thermal glass constants for glasses supplied by Schott have been calculated, which are based on CTE and the variation of the refractive index with temperature [11-16].

Figure 1 shows the athermal glass map for visible Schott

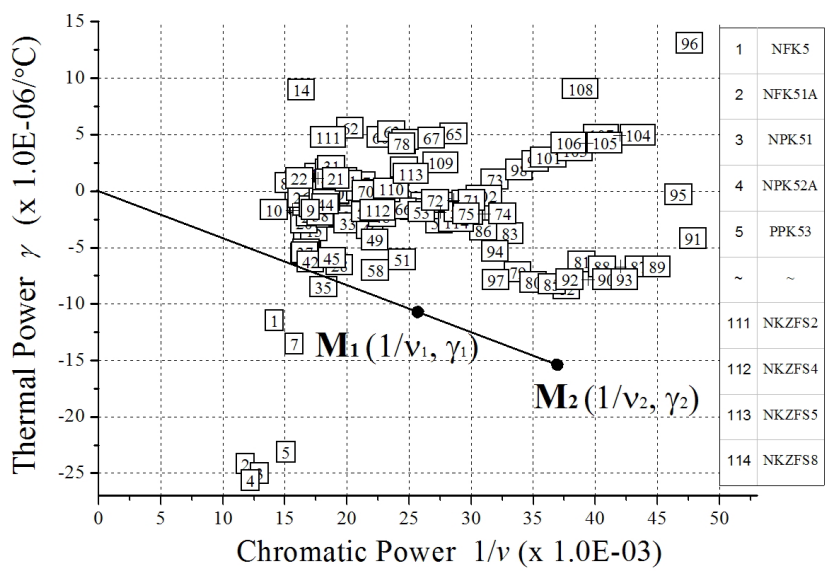

FIG. 1. Athermal glass map plotting $\gamma$ v.s. $1 / v$ for visible Schott glasses.

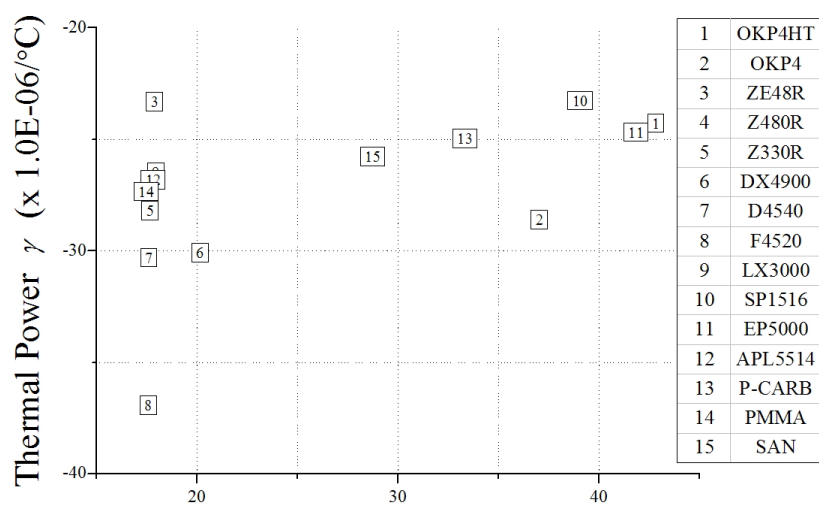

Chromatic Power $1 / v(\mathrm{x} 1.0 \mathrm{E}-03)$

FIG. 2. Athermal glass map plotting $\gamma$ v.s. $1 / v$ for visible optical plastics. 


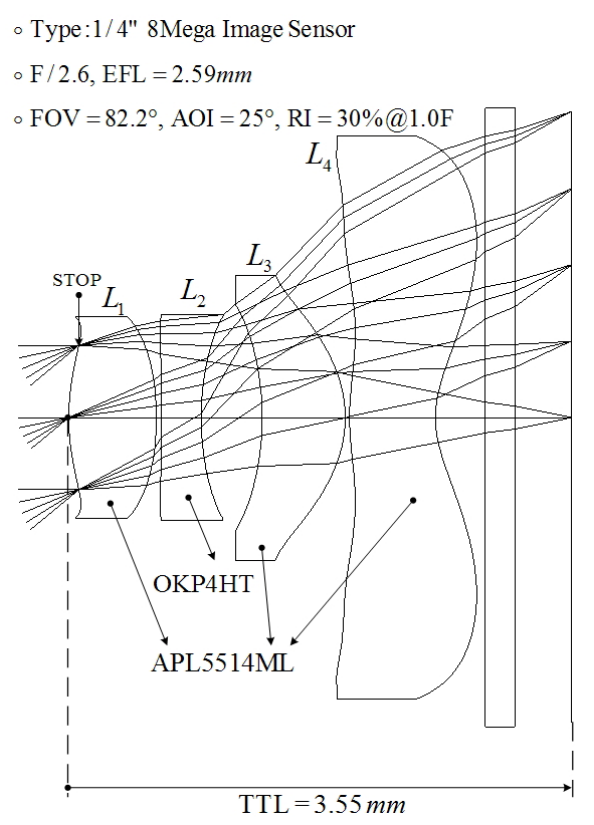

FIG. 3. Layout of a starting lens for phone camera.

glass. In Fig. 1, inverse Abbe number(1/v) is designated as a chromatic power on the horizontal axis, and thermal glass $\operatorname{constant}(\gamma)$ as a thermal power on the vertical axis. Figure 2 illustrates the athermal glass map for 15 optical plastics that are widely used in mobile cameras [17].

By plotting the thermal glass constant versus the inverse Abbe number, we can visually identify two materials that satisfy the athermal and achromatic conditions given by Eq. (6). In Fig. 1, any two materials $\left(\mathrm{M}_{1}, \mathrm{M}_{2}\right)$ that can be connected by a line that passes through the origin will provide athermal and achromatic solutions. In other words, if one material is known on an athermal glass map, like $\mathrm{M}_{1}\left(1 / v_{1}, \gamma_{1}\right)$, then another material $\left(\mathrm{M}_{2}\right)$ that serves color correction and passive athermalization can be found on a line connecting two materials. Even if a lens system is composed of many elements shown in Fig. 3, the equivalent Abbe number and equivalent thermal glass constant reduce the problem to be that of a two-element system as a doublet. Unlike some of the previous methods using complex triangle geometry $[7,8]$, we can use this method to visually determine exactly what material is required to achromatize and athermalize the multi-lens system.

\section{ATHERMAL AND ACHROMATIC DESIGN FOR A PHONE CAMERA LENS USING GRAPHICAL SELECTION OF GLASS PAIR}

\subsection{Analysis of a Starting Design}

A phone camera lens composed of four plastics, equipped with a quarter inch image sensor having 8-mega pixels, is given as a starting case, as shown in Fig. 3. This lens has great thermal defocuses, as illustrated in Fig. 4, in

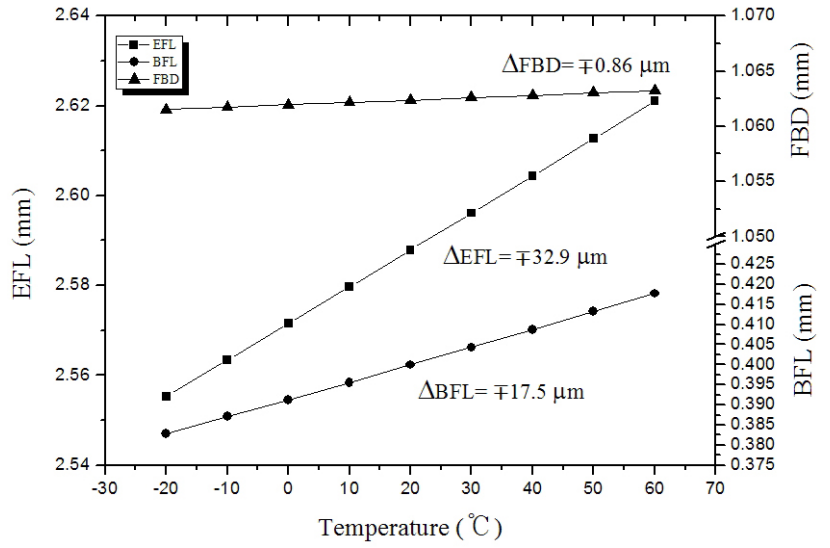

FIG. 4. Thermal defocuses of a starting lens for effective focal length(EFL), back focal length(BFL), and flange back distance(FBD).

particular, the variations for effective focal length $(\triangle \mathrm{EFL})$ over $-20^{\circ} \mathrm{C}$ to $+60^{\circ} \mathrm{C}$ are $\mp 32.9 \mu \mathrm{m}$. However, note that the variations of flange back distance $(\triangle \mathrm{FBD})$, depending on the thermal expansion and the length of a housing material which is of AL6061, is just $\mp 0.86 \mu \mathrm{m}$. This quantity is so small that the thermal defocus by the housing would be negligible, as pointed out in Section III. The starting lens fulfills many requirements of a current mobile phone camera, except color correction and stable thermal focus. In this research, however, the achromatic and passively athermalized design will be discussed using graphical selection of the materials.

\subsection{Graphical Selection of a Material Pair and Optimized Design}

In a phone camera lens with four elements, three elements of $\mathrm{L}_{2}, \mathrm{~L}_{3}$, and $\mathrm{L}_{4}$ are chosen such that they create an equivalent triplet, with equivalent Abbe number $\left(v_{234}\right)$ and equivalent thermal glass constant $\left(\gamma_{234}\right)$. This equivalent triplet then can be used in combination with another element $\left(L_{1}\right)$ to realize an athermal and achromatic lens system. The equivalent Abbe number and equivalent thermal glass constant are calculated from Eqs. (12) and (15):

$$
\begin{gathered}
v_{234}=\frac{\phi_{2}+\phi_{3}+\phi_{4}}{\left(\phi_{2} / v_{2}\right)+\left(\phi_{3} / v_{3}\right)+\left(\phi_{4} / v_{4}\right)}=22.06, \\
\gamma_{234}=\frac{\gamma_{2} \phi_{2}+\gamma_{3} \phi_{3}+\gamma_{4} \phi_{4}}{\phi_{2}+\phi_{3}+\phi_{4}}=-245.668 \times 10^{-6} /{ }^{\circ} \mathrm{C} .
\end{gathered}
$$

The equivalent triplet having $v_{234}=22.06$ and $\gamma_{234}=$ $-245.668 \times 10^{-6} /{ }^{\circ} \mathrm{C}$ is located at $\mathrm{M}_{\mathrm{e}}$ in Fig. 5. By connecting this point $\left(\mathrm{M}_{\mathrm{e}}\right)$ to the origin with a line, the material of the first lens $\left(\mathrm{L}_{1}\right)$ can be selected from glasses being on this line, satisfying both athermal and achromatic conditions. From case 1 of Fig. 5, the glass of the first lens is graphically 


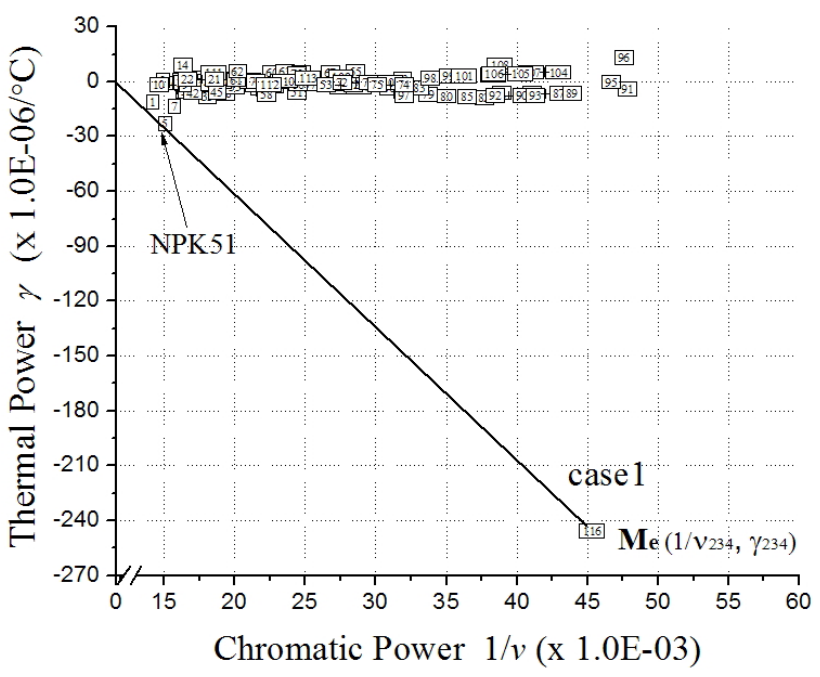

FIG. 5. Graphical selection of a glass material from the equivalent triplet on athermal glass map.

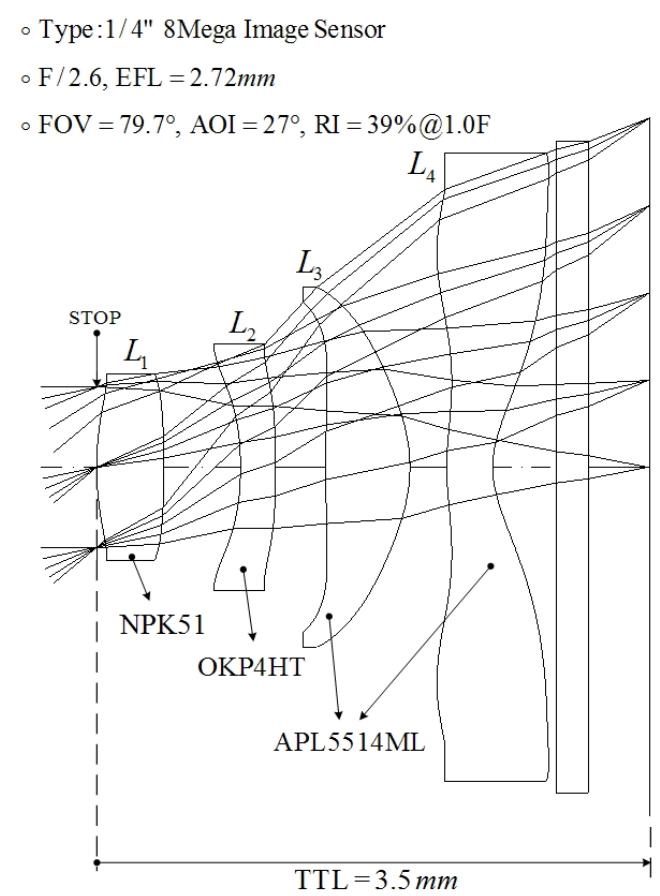

FIG. 6. Layout of an athermal and achromatic lens for phone camera.

selected to be NPK51 of Schott. This material combination also satisfies the Eq. (6). The other good solution is not found from different combinations for materials, for example, $\mathrm{L}_{2}$ versus $\mathrm{L}_{134}, \mathrm{~L}_{3}$ versus $\mathrm{L}_{124}, \mathrm{~L}_{4}$ versus $\mathrm{L}_{123}$. In Eqs. (17) and (18), the triplet assumes the thin lenses in contact, however, this approach allows for a good starting point.

After replacing the first lens glass into NPK51 and bending, the starting lens has been re-optimized using Code-V. Finally, a lens for a phone camera having good performance is obtained, as shown in Fig. 6. This lens has small chromatic

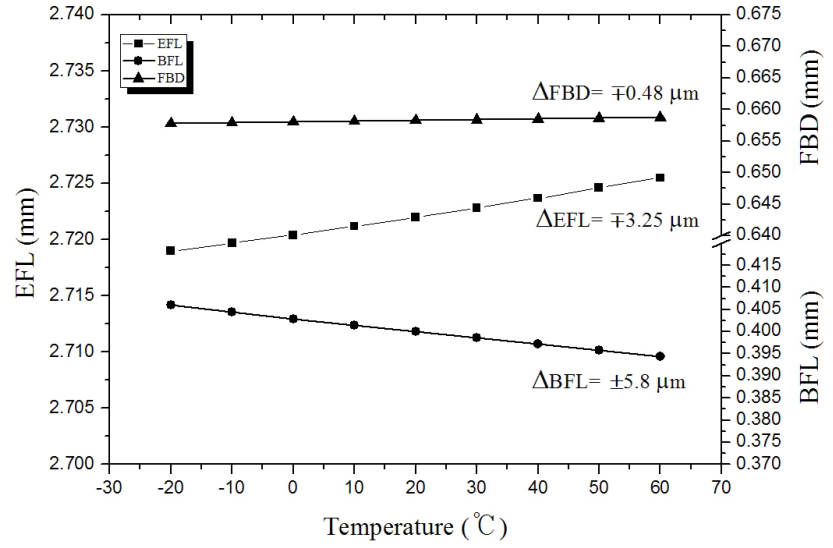

FIG. 7. Thermal defocuses of an athermal and achromatic lens for effective focal length(EFL), back focal length(BFL), and flange back distance(FBD).

defocus less than $\pm 10 \mu \mathrm{m}$ between $\mathrm{C}$ - and F-lines. The thermal defocuses of this lens are significantly reduced, as illustrated in Fig. 7. The variations for effective focal length and back focal length over $-20^{\circ} \mathrm{C}$ to $+60^{\circ} \mathrm{C}$ are $\mp 3.25 \mu \mathrm{m}$ and $\pm 5.8 \mu \mathrm{m}$, respectively. Also, the variation of flange back distance is just $\mp 0.48 \mu \mathrm{m}$, which is much less than the quarter-wave depth of focus. This quantity is so small that the thermal defocus by the housing would be negligible. Thus, all thermal defocuses are much less than the depth of focus $(\delta)$ given by Eq. (19):

$$
\delta= \pm \frac{\mathrm{f}-\text { number }}{\text { maximum frequency }}= \pm 11.8 \mu \mathrm{m}
$$

In conclusion, the designed lens is achromatic in visible light and passively athermalized over $-20^{\circ} \mathrm{C}$ to $+60^{\circ} \mathrm{C}$.

Figure 8 shows the modulation transfer function (MTF) characteristics of two lenses with temperature. The MTF of a designed lens from athermal and achromatic processes is much more stable than that of a starting lens over the specified range of temperature and more than $50 \%$ at $110 \mathrm{lp} / \mathrm{mm}$ over most fields. Also, the MTF at maximum frequency of $220 \mathrm{lp} / \mathrm{mm}$ is more than $30 \%$ except for a marginal field, the ratio of relative illuminations is more than $39 \%$ over all fields, and the distortion is less than $\pm 1 \%$. The finally designed lens for phone camera has an f-number of 2.6, focal length of $2.72 \mathrm{~mm}$, and good performances including stable thermal focusing. Consequently, this lens has enough performance to fulfill the requirements of a current mobile phone camera.

\section{CONCLUSION}

By plotting the thermal glass constant versus the inverse Abbe number, athermal glass maps were derived for Schott glasses and plastic materials in visible light. For a lens 


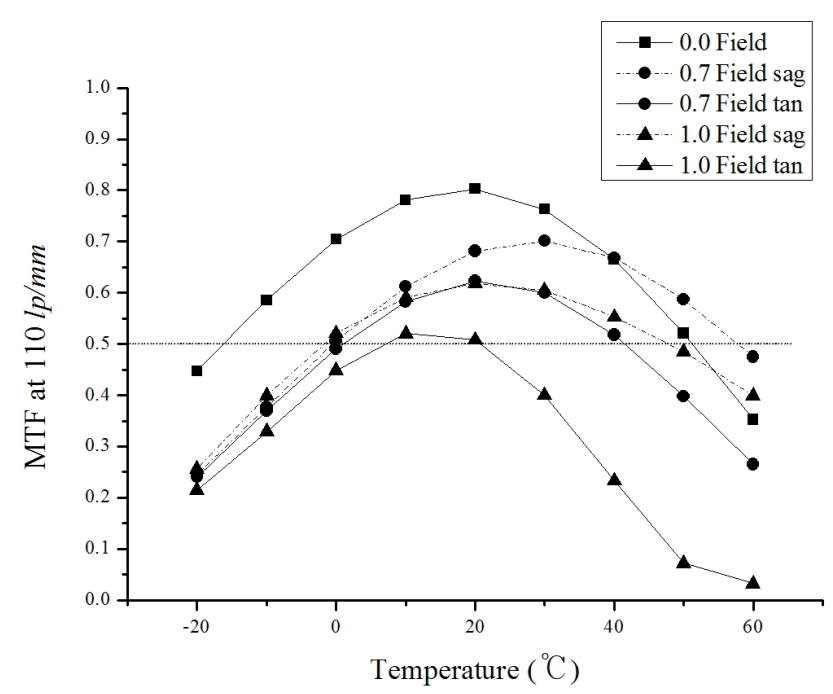

(a)

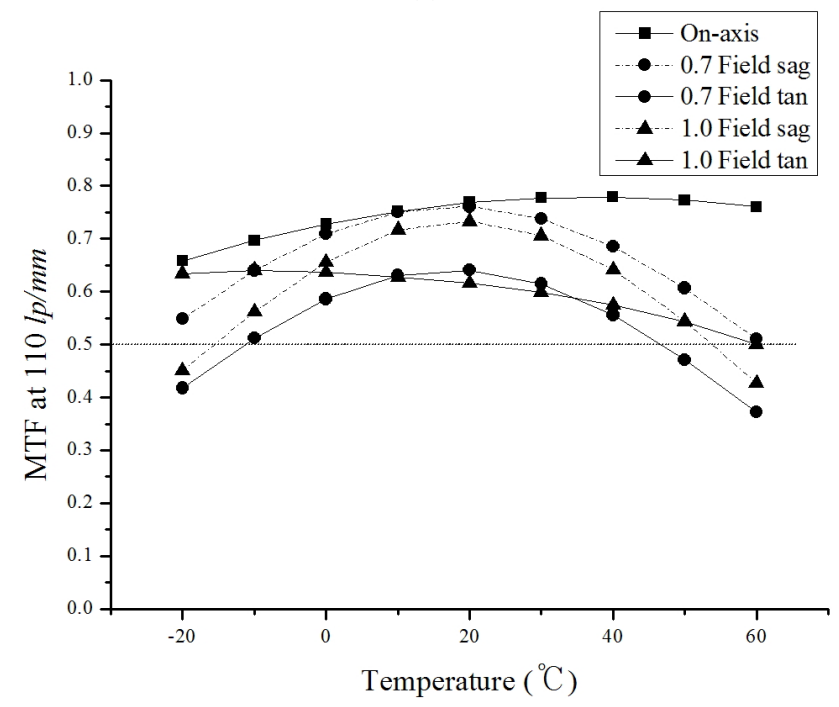

(b)

FIG. 8. A comparison of the MTF at $110 \mathrm{lp} / \mathrm{mm}$ with temperature. (a) Starting lens, (b) athermal and achromatic lens.

system composed of many elements, we suggested the method of obtaining the equivalent Abbe number and equivalent thermal glass constant. By selecting the corresponding materials for an equivalent lens on an athermal glass map, we can simply identify a pair of materials that satisfy the athermal and achromatic conditions. This graphical method of selecting materials is a simple and powerful way to find a design solution. By utilizing the graphs and the concept of equivalence presented in this study, a good solution for a phone camera lens has been found. The finally designed lens has an f-number of 2.6, focal length of $2.72 \mathrm{~mm}$, and good performances including stable thermal focusing. The expansion of an athermal glass map into a multi-lens system spaced element by element will be discussed in a following paper.

\section{REFERENCES}

1. J. W. Perry, J. Johnson, J. H. Jeffree, D. S. Grey, and D. S. Volosov, "Fundamentals, techniques and design," in Handbook of Optics, 2nd ed., M. Bass, ed. (McGraw-Hill, New York, USA, 1995), vol. 1.

2. H. Jamieson, "Thermal effects in optical systems," Opt. Eng. 20, 156-160 (1981).

3. L. Friedman, "Thermo-optical analysis of two long-focallength aerial reconnaissance lenses," Opt. Eng. 20, 161-165 (1981).

4. G. Kurbitz, "Automatic active athermalization of infrared optical systems," Proc. SPIE 1540, 612-621 (1991).

5. P. J. Rogers, “Athermalized FLIR optics," Proc. SPIE 1354, 742-751 (1990).

6. L. Rayces and L. Lebich, "Thermal compensation of infrared achromatic objectives with three optical materials," Proc. SPIE 1354, 752-759 (1990).

7. Y. Tamagawa, S. Wakabayashi, T. Tajime, and T. Hashimoto, "Multilens system design with an athermal chart," Appl. Opt. 33, 8009-8013 (1994).

8. Y. Tamagawa and T. Tajime, "Expansion of an athermal chart into a multilens system with thick lenses spaced apart," Opt. Eng. 35, 3001-3006 (1996).

9. Povey, "Athermalization techniques in infrared systems," Proc. SPIE 655, 142-153 (1986).

10. R. M. Hudyma, "Athermal MWIR objectives," Proc. SPIE 2540, 229-235 (1995).

11. Schott, Optical Glass Catalogue Excel (Schott Inc., Germany, June, 2012).

12. Schott, "TIE-19: Temperature coefficient of the refractive index," in Proc. Schott Technical Information (Schott Inc., Germany, July, 2008).

13. B. Edlen, "The dispersion of standard air," J. Opt. Soc. Am. 43, 339-344 (1953).

14. E. R. Peck and K. Reeder, "Dispersion of air," J. Opt. Soc. Am. 62, 958-962 (1972).

15. P. E. Ciddor, "Refractive index of air: New equations for the visible and near infrared," Appl. Opt. 35, 1566-1573 (1996).

16. P. R. Yoder, Mounting Optics In Optical Instruments (SPIE Press, Bellingham, USA, 2002), Chapter 12.

17. S. Baumer, Handbook of Plastic Optics (WLEY-VCH, Germany, 2010), Chapter 5. 